\title{
Intermittent thermal plasma acceleration linked to sporadic motions of the magnetopause, first Cluster results
}

\author{
J.-A. Sauvaud ${ }^{1}$, R. Lundin ${ }^{1,2}$, H. Rème ${ }^{1}$, J. P. McFadden ${ }^{3}$, C. Carlson ${ }^{3}$, G. K. Parks ${ }^{3}$, E. Möbius ${ }^{4}$, L. M. Kistler ${ }^{4}$, \\ B. Klecker ${ }^{5}$, E. Amata ${ }^{6}$, A. M. DiLellis ${ }^{6}$, V. Formisano ${ }^{6}$, J. M. Bosqued ${ }^{1}$, I. Dandouras ${ }^{1}$, P. Décréau ${ }^{7}$, M. Dunlop ${ }^{8}$, \\ L. Eliasson ${ }^{2}$, A. Korth ${ }^{9}$, B. Lavraud ${ }^{1}$, and M. McCarthy ${ }^{10}$ \\ ${ }^{1}$ CESR, Toulouse, France \\ ${ }^{2}$ Institut for Rymdfysik, Kiruna, Sweden \\ ${ }^{3}$ Space Sciences Laboratory, University of California, Berkeley, USA \\ ${ }^{4}$ EOS, University of New Hampshire, Durham, NH, USA \\ ${ }^{5}$ MPI für extraterrestrische Physik, Garching, Germany \\ ${ }^{6}$ IFSI, Rome, Italy \\ ${ }^{7}$ LPCE, Orléans, France \\ ${ }^{8}$ Imperial College, London, UK \\ ${ }^{9}$ MPI für Aeronomie, Katlenburg-Lindau, Germany \\ ${ }^{10}$ Space Program, University of Washington, USA
}

Received: 2 May 2001 - Revised: 20 July 2001 - Accepted: 28 August 2001

\begin{abstract}
This paper presents the first observations with Cluster of a very dense population of thermal ionospheric ions $\left(\mathrm{H}^{+}, \mathrm{He}^{+}, \mathrm{O}^{+}\right)$locally "accelerated" perpendicularly to the local magnetic field in a region adjacent to the magnetopause and on its magnetospheric side. The observation periods follow a long period of very weak magnetic activity. Recurrent motions of the magnetopause are, in the presented cases, unexpectedly associated with the appearance inside closed field lines of recurrent energy structures of ionospheric ions with energies in the $5 \mathrm{eV}$ to $\sim 1000 \mathrm{eV}$ range. The heaviest ions were detected with the highest energies. Here, the ion behaviour is interpreted as resulting from local electric field enhancements/decreases which adiabatically enhance/lower the bulk energy of a local dense thermal ion population. This drift effect, which is directly linked to magnetopause motions caused by pressure changes, allows for the thermal ions to overcome the satellite potential and be detected by the suprathermal CIS Cluster experiment. When fast flowing, i.e. when detectable, the density $\left(\sim 1 \mathrm{~cm}^{-3}\right)$ of these ions from a terrestrial origin is (in the cases presented here) largely higher than the local density of ions from magnetospheric/plasma sheet origin which poses again the question of the relative importance of solar and ionospheric sources for the magnetospheric plasma even during very quiet magnetic conditions.
\end{abstract}

Key words. Ionosphere (planetary ionosphere; plasma con-

Correspondence to: J.-A. Sauvaud (sauvaud@ cesr.fr) vection) Magnetospheric physics (magnetopause, cusp and boundary layers)

\section{Introduction}

Ions of terrestrial origin are systematically expelled from the polar regions over a wide energy range from suprathermal energies in the polar wind up to several $\mathrm{keV}$ over the auroral regions (see a review by Yau and André, 1997). The magnetospheric dynamics of these ionospheric ions strongly depend on their initial parallel energy. For ions with very low energy, the convection drift is dominant. They do not bounce, but locally $\boldsymbol{E} \times \boldsymbol{B}$ drift even up to distant regions of the magnetosphere (e.g. Sauvaud and Delcourt, 1987; Delcourt et al., 1989, 1993). When projected along the field lines, the ion trajectories simply follow the ionospheric convection cells. For a two-cell convection pattern, slightly suprathermal ions escaping from the high-latitude regions are $\boldsymbol{E} \times \boldsymbol{B}$ drifting to the dayside. It must be stressed that in the distant magnetosphere, the plasma $\boldsymbol{E} \times \boldsymbol{B}$ drift is much larger than the equivalent ionospheric convection. In a first approximation, the electric field, $E$, varies approximately as $B^{1 / 2}$, which means that during periods of strongly enhanced convection, i.e. 0.3$0.5 \mathrm{~km} / \mathrm{s}$, at ionospheric altitudes, $\mathrm{O}^{+}$ions will have a drift energy of the order of $15 \mathrm{eV}-50 \mathrm{eV}$ in the distant magnetosphere. This is well within the energy range of the Cluster 
CIS instruments and above the satellite potential of about 10 volts in the magnetosphere (plasma sheet).

The plasmasphere constitutes another potential reservoir of terrestrial plasma. Recent works by Su et al., (2000, 2001) have demonstrated that geomagnetic activity increases bring unusually high densities of plasmaspheric material to the dayside magnetopause, as parts of the outer plasmasphere are swept away by increased convection (e.g. Chen et al., 1975). Furthermore, using an imaging device at $304 \AA$, with a wavelength emitted by helium ions at the Sun and resonantly scattered by plasmaspheric helium ions, the IMAGE mission has recently performed the first remote observations of such plumes in connection with activity increases (Burch et al., 2001).

After the discovery of ionospheric ion escape into the magnetosphere (Shelley et al., 1976), the consensus view is that the ionosphere is an important, if not dominant source of magnetospheric plasma (e.g. Chappell et al., 1987). However, only a part of the terrestrial ion distribution has been properly investigated within the magnetosphere. Mass spectrometer flown in the outer magnetosphere have so far only been able to measure a limited portion of the total solid angle. These limitations have recently been overcome in the frame of the Fast, Polar, Equator-S and Cluster projects where the ion mass and energy distribution functions are measured over $\sim 4 \pi$ (see Moore et al., 1995; Shelley et al., 1995; Rème et al., 1997, 2001; Möbius et al., 1998; Carlson and McFadden, 1998; McFadden and Carlson, 1998). Another limitation was the uncontrolled positive satellite potential. For the Polar and Cluster projects the satellite electric potential was reduced using respectively a plasma source (Moore et al., 1995) and an ion emitter (Riedler et al., 1997). However, the spacecraft potential still reaches several volts in the outer dayside magnetosphere (outside the dense cusp) which precludes the direct measurement here of very cold and non-flowing terrestrial plasma by using particle detectors.

The first observation indicating that cold plasma may become "visible" in the immediate vicinity of the magnetopause supposedly by an enhanced convection electric field was presented by Lundin and Dubinin (1985). However, the energy, and the angular and time resolution of their data was insufficient to resolve the properties of this acceleration/convection region. Fuselier (1989) reported evidences for two distinct $\mathrm{O}^{+}$populations in the dayside sub-solar LLBL: (i) $\mathrm{O}^{+}$convected into the boundary layer from the plasma sheet with $E>6 \mathrm{keV}$, and (ii) new field-aligned $\mathrm{O}^{+}$ beams $(E>100 \mathrm{eV})$ injected directly from the high-latitude ionosphere. Recently, Chandler et al. (1999) reported from the Polar TIDE experiment the detection of a cold plasma population $(T<2 \mathrm{eV})$ flowing along the field lines with a parallel velocity of the order of $50 \mathrm{~km} / \mathrm{s}$ in the outer magnetosphere. These authors propose that cold ionospheric ions with initially slow field-aligned speeds were accelerated upon reflection from the magnetopause.

In this paper, we present first the detailed measurements of a dense $\left(\sim 1 \mathrm{~cm}^{-3}\right)$ population of low energy ionospheric ions, $\mathrm{H}^{+}, \mathrm{He}^{+}, \mathrm{O}^{+}$, detected during very quiet time periods
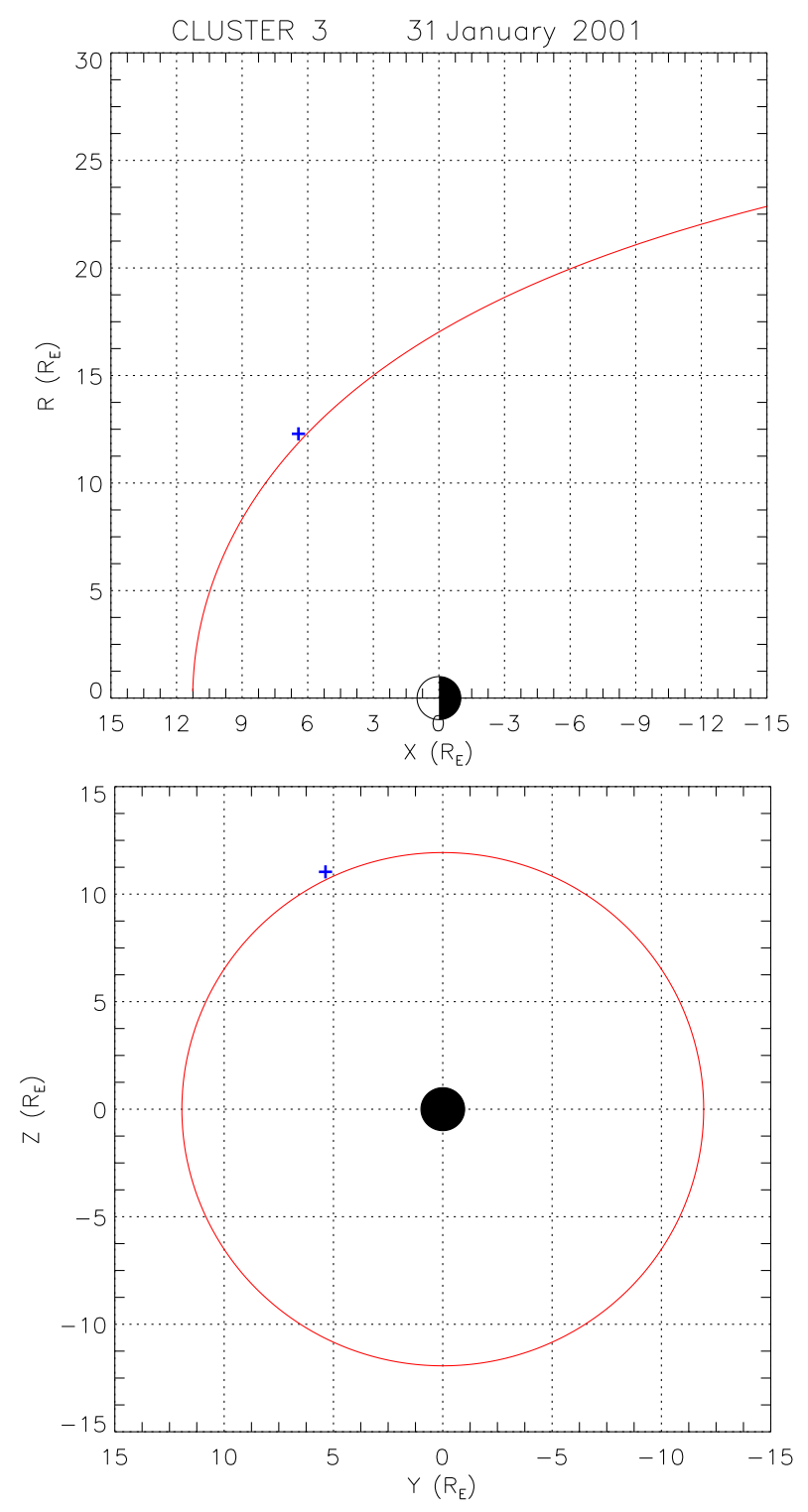

Fig. 1. Cluster location in a GSE coordinate system on 31 January 2001 around 05:45 UT. Top panel: projection in a $X$, $R=\left(X^{2}+Y^{2}\right)^{1 / 2}$ frame. Bottom panel: projection in the $(Y$, $Z)$ plane.

in a layer adjacent to the magnetopause. We show that intermittent motions of the magnetopause are associated with the appearance of these terrestrial ions drifting perpendicularly to the magnetic field with velocities corresponding to energies in the $\sim 10 \mathrm{eV}$ to $1000 \mathrm{eV}$ range, depending on the species. Here, the ion behaviour is interpreted as a result from local electric field enhancements linked to magnetopause motions that are caused by pressure changes. These motions give intermittently enough energy to a local, hidden, dense thermal ion population, slowly convected there along the magnetic field, to overcome the satellite potential and be detected by the suprathermal CIS experiment on board Cluster. 

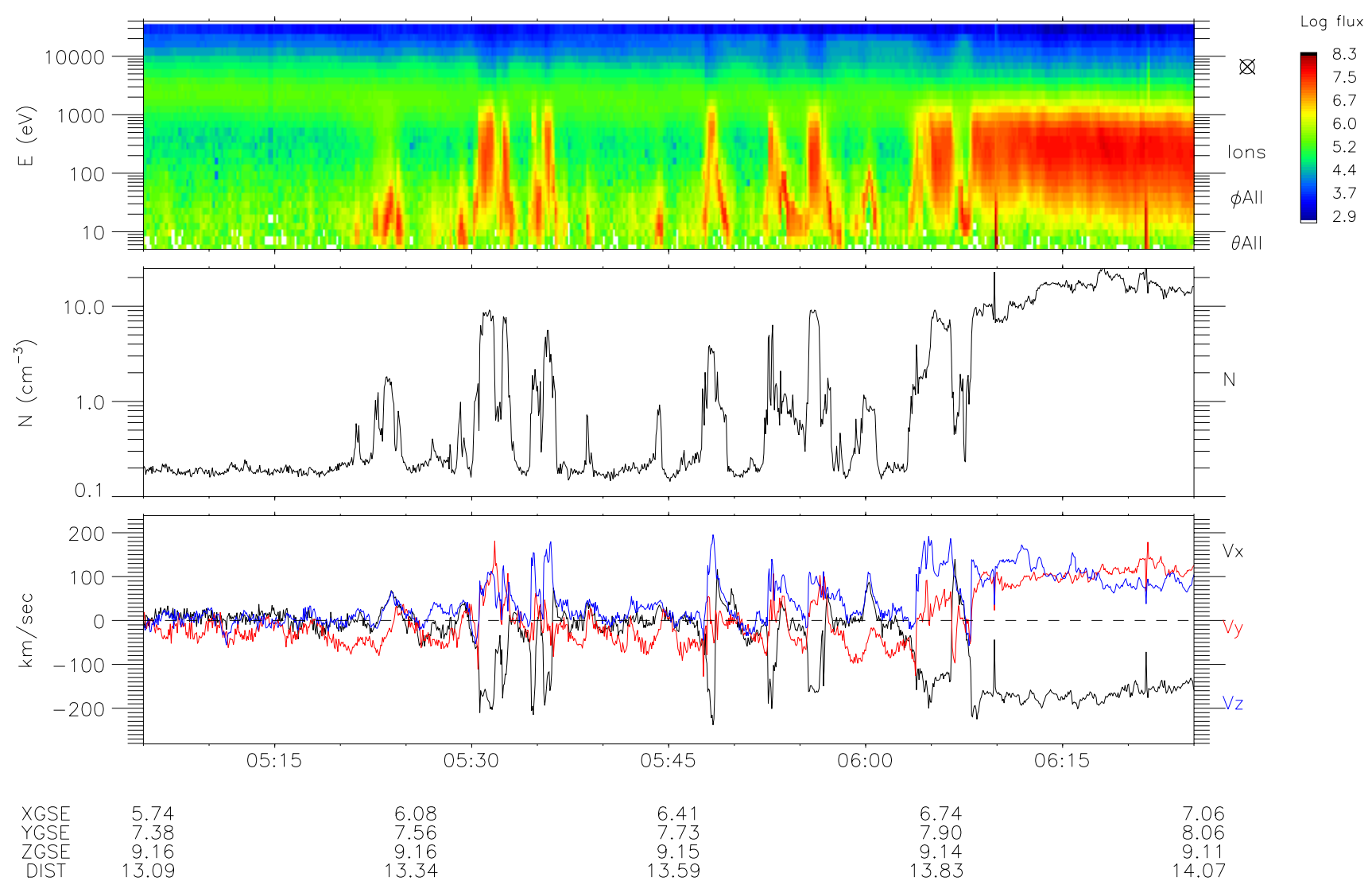

Fig. 2. Overview of the ion measurements (without mass resolution) on board Cluster \#3 on 31 January 2001 between 05:05 and 06:25 UT. From top to bottom: ion energy-time spectrogram with color-coded fluxes, $\left(\mathrm{cm}^{2} \text {.s.ster.keV }\right)^{-1}$; density and the three GSE components of the ion velocity vector. The moments are computed on board.

\section{Data sources}

The particle data used in this paper come from the Cluster Ion Spectrometer (CIS) experiment which comprises (Rème et al., 1997; this issue): (i) a Hot Ion Analyser, CIS-2, measuring the ion distributions from $5 \mathrm{eV}$ to $26 \mathrm{keV}$ by combining a classical symmetrical quadrispherical analyser with a fast particle imaging system based on microchannel plate electron multipliers and position encoding discrete anodes (Carlson et al., 1982); (ii) a time-of-flight mass spectrometer, CIS-1, which combines a top-hat analyser with an instantaneous $360^{\circ} \times 8^{\circ}$ field-of-view with a time-of-flight section to measure complete 3D distribution functions of the major ion species. Typically, these include $\mathrm{H}^{+}, \mathrm{He}^{++}, \mathrm{He}^{+}$and $\mathrm{O}^{+}$. The sensor primarily covers the energy range between 0.02 and $38 \mathrm{keV} / \mathrm{q}$. The magnetic field data used in this paper comes from the fluxgate magnetometers (FGM) installed on board the Cluster spacecraft (Balogh et al., 1997). These data are averaged over $1 \mathrm{~s}$. For the 31 January 2001 case presented in detail in this paper, the ion emitter experiment ASPOC was off; the satellite potential varied between +10 to +15 volts inside the magnetosphere and reached about 4-6 volts in the magnetosheath. We also present data corresponding to a similar observation on 16 February 2001 when the ASPOC experiment was on, thus keeping the satellite potential (spacecraft 3) below 7 volts.

\section{Observations}

Figure 1 presents the position of the four Cluster satellites for the first period of interest on 31 January 2001, around 05:45 UT in a GSE coordinate system. The magnetopause model is that given by Shue et al. (1997). It has been adjusted to take into account the solar wind conditions provided by the ACE satellite. (A time delay of $68 \mathrm{~min}$ has been applied for the solar wind travel time between ACE and the Earth). Cluster was located at high-latitudes on the dusk side, very close to the expected position of the magnetopause. The IMF $B_{X}$ was largely stronger than the $B_{Y}$ component, i.e. the bow shock was quasi-parallel near the sub-solar point which is known to favor the generation of Pc-3/5 waves into the magnetosphere in association with magnetosheath pressure pulses. The IMF $B_{Z}$ component oscillates between small positive and negative values until $\sim$ 04:30 UT when it turned 


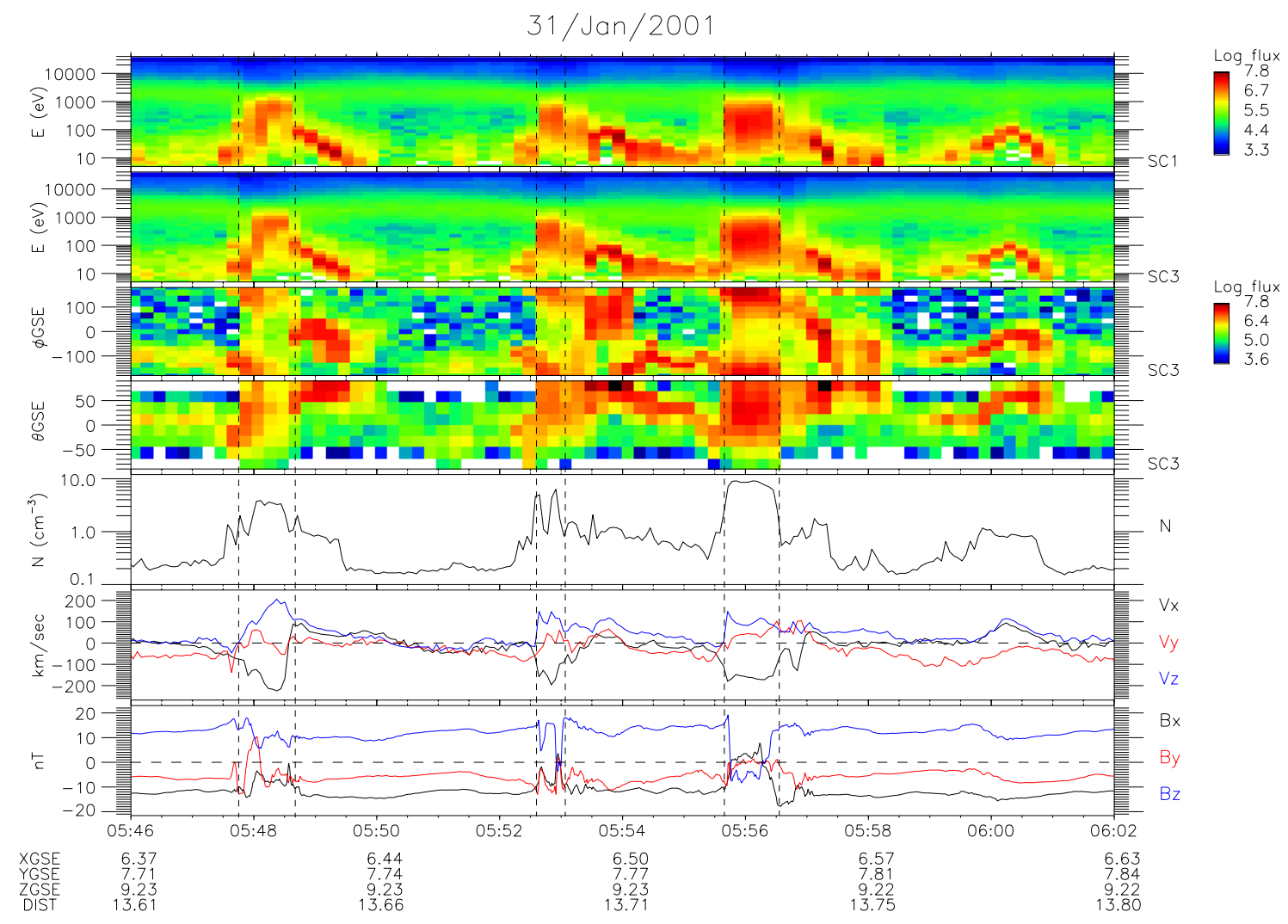

Fig. 3. A close-up of the ion and magnetic field measurements performed between 05:46 and 06:02 UT on 10 January 2001 during several encounters with the magnetosheath. The two top panels give the CIS-2 ion energy-time spectrograms for Cluster 1 and 3 . The time resolution is 12. The two following panels display the ion anisotropy measured between 5 and $300 \mathrm{eV}$ on board Cluster 3 in the spin plane ( $\phi \mathrm{GSE})$ and in a rotating plane containing the spin axis $(\theta \mathrm{GSE})$. The fifth and sixth panels give the ion density and the three GSE components of the ion bulk velocity. The last panel displays the three GSE components of the magnetic field vector.

clearly negative. It must be stressed that the magnetic activity was extremely weak during the Cluster measurements and during the 20 preceding hours (the provisional AE stayed below $\sim 50 \mathrm{nT}$ ).

We will also present data pertaining to 16 February 2001 which is also a day corresponding to weak magnetic activity. AE was lower than $50 \mathrm{nT}$ during the measurements and the IMF $B_{X}$ component was nearly constant and of the order of $3 \mathrm{nT}$, while the IMF $B_{Z}$ and $B_{Y}$ components were close to zero. For that event, the Cluster position $\left(X_{\mathrm{GSE}}=5.4 R_{E}, Y_{\mathrm{GSE}}=4.9 R_{E}, Z_{\mathrm{GSE}}=9.0 R_{E}\right)$ was quite similar to that for 31 January 2001 (see Fig. 1).

Figure 2 presents an overview of the Cluster pass from the dayside plasma sheet to the magnetosheath, between 05:05 and 06:25 UT on 31 January 2001. The upper panel of the figure gives the omnidirectional ion energy-time spectrogram provided by the CIS-2 instrument on board Cluster 3 . The middle panel shows the onboard computed ion density and the bottom panel provides the three GSE components of the plasma velocity. On that day, the Cluster spacecraft definitively entered the magnetosheath at $\sim 06: 08$ UT. Starting around 05:30 UT, Cluster crossed several times the magnetopause and spent several minutes each time in the magnetosheath, detecting the dense and fast flowing plasma of the solar wind origin, before entering back into the magnetosphere. Such magnetosheath encounters correspond, as expected, to strong anti-sunward plasma velocities (higher than about $150 \mathrm{~km} / \mathrm{s}$ ). Beginning at $\sim 05: 22 \mathrm{UT}$, the spacecraft also detected intermittently a dense and low energy plasma $(E<300 \mathrm{eV})$ with a bulk flow velocity lower than inside the magnetosheath. Encounters with such a low energy ion population can be clearly recognized at 05:22, 05:28, 05:38, 05:44, 06:00 UT.

Figure 3 presents detailed particles and B field data obtained onboard Cluster 1 and 3 between 05:46 and 06:02 UT on 31 January 2001, when the four Cluster spacecraft crossed three times into the magnetosheath and back into the magnetosphere (indicated by vertical dashed lines at $\sim 05: 47: 45$, 00:52:30 and 00:55:32 UT). ACE data indicate variations in the solar wind dynamical pressure by about $25 \%$ during this time interval (not shown). However, we do not exclude the possibility that oscillations of the magnetopause can be due to sporadic or time-dependant reconnection. The small satellite separation $(<600 \mathrm{~km})$ coupled to the CIS time resolution $(4 \mathrm{~s})$ and the high velocity of the magnetopause $(>100 \mathrm{~km} / \mathrm{s})$ does not allow one to study the details of the magnetopause motion. The two top panels of Fig. 3 display the energytime spectrograms (Cluster 1 and 3) of ions without mass 


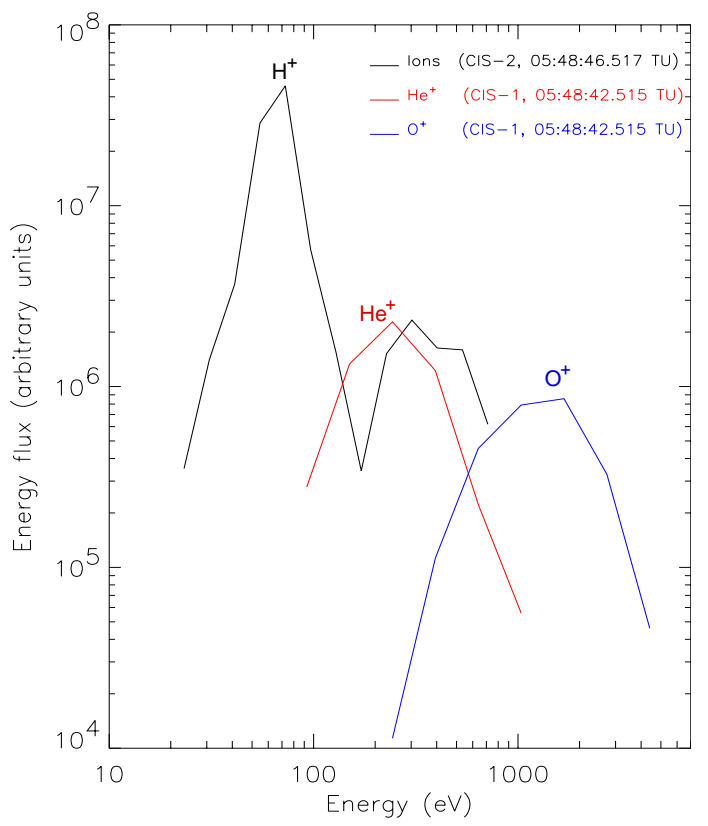

Fig. 4. Ion energy spectra from CIS-2 (averaged on board over $12 \mathrm{~s}$ ) and energy spectra for $\mathrm{He}^{+}$and $\mathrm{O}^{+}$from the mass spectrometer CIS-1 (averaged on board over $32 \mathrm{~s}$ ). Data are taken in a low energy structure encountered inside the magnetosphere, after the satellite exits from the magnetosheath at $\sim 05: 48: 20$ UT (see Fig. 3).

separation in the full CIS-2 energy range of $5 \mathrm{eV}$ to $26 \mathrm{keV}$. The spectrograms are constructed from the 3D distribution of the ions. We used here the best available time resolution for 3D distributions at that time $(12 \mathrm{~s})$. Energy fluxes are color-coded. The plasma sheet population is seen as the green area with energies on the order of $1-8 \mathrm{keV}$. The magnetosheath is seen in the range of $200 \mathrm{eV}$ to $1 \mathrm{keV}$ and the low energy population discussed in this paper appears as energy dispersed signatures with an energy generally between 5 and $<300 \mathrm{eV}$. The third and fourth panels of Fig. 3, respectively, give the phase and polar angle distribution of ion fluxes with energies between 5 and $300 \mathrm{eV}$. For example, ions measured during the third magnetosheath encounter (05:55:37 to 05:56:03 UT) were flowing in the direction of phase angles close to $180^{\circ}$ and $-180^{\circ}$, that is, coming from the sunward direction with a broad polar angle range higher than $50^{\circ}$ toward the $+Z_{\mathrm{GSE}}$ axis. These directions are consistent with particles traveling, as expected, from the magnetosheath at high-latitudes in the dusk sector. Here, the energy range of $5 \mathrm{eV}-300 \mathrm{eV}$ has been chosen for display to illustrate the dynamics of the low energy ions. The fifth panel displays the ion density measured on board satellite 3 in the full energy range with a resolution of $4 \mathrm{~s}$ (computed onboard). The sixth panel shows the three components of the ion bulk velocity computed onboard the same satellite and the bottom panel shows the three components of the magnetic field. The magnetopause encounters can be identified from gradients in the ion velocity components and in the $B$ field components.

As a general trend, just before each exit of the satellite into
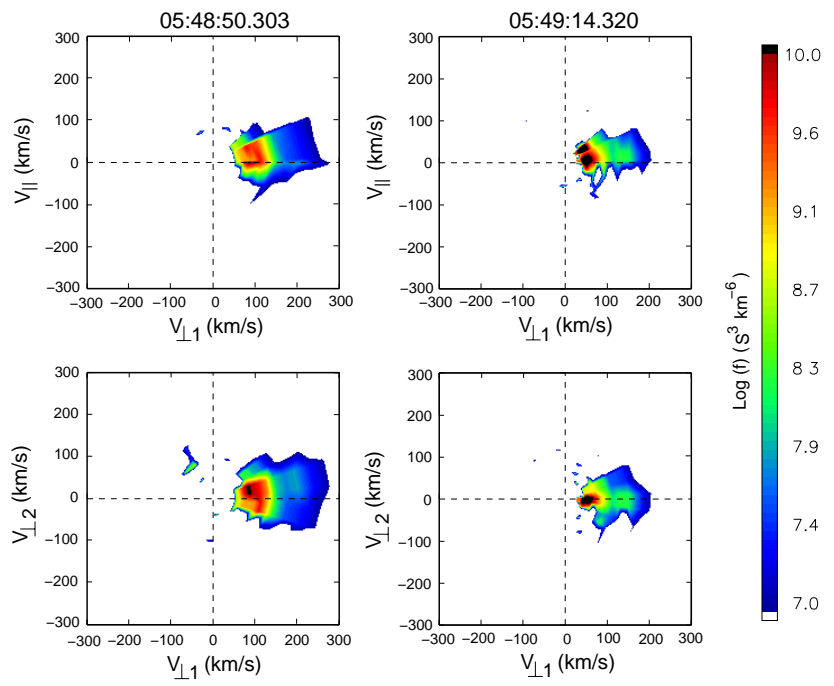

Fig. 5. Top: ion distribution function in a plane $\left(V_{/ /}, V_{\perp}\right)$. Bottom: in a $\left(V_{\perp 1}, V_{\perp 2}\right)$ plane. The distributions are averaged on board over $12 \mathrm{~s}$. The $\left(V_{/ /}, V_{\perp}\right)$ plane contains the onboard computed bulk velocity. Left, distribution starting at 05:48:50.303. Right, starting at 05:49:14.320 (see text and Fig. 3). These data come from the CIS-2 sensor (no mass resolution).

the magnetosheath, low energy ions form a ramp observable at energies in the $5 \mathrm{eV}$ to $\sim 100 \mathrm{eV}$ range. When the satellites re-enter the magnetosphere, they again encounter low energy ions showing a clear energy dispersion for about one minute; the energy is decreasing with time. As we will show below, this energy dispersion is apparently due to a change in bulk velocity. These structures are clearly located on closed field lines. This can be appreciated from the values of the components of the magnetic field. Inside these structures, the ion density reaches about $1 \mathrm{~cm}^{-3}$ which contrasts with the density inside the plasma sheet which here does not exceed 0.2 $0.3 \mathrm{~cm}^{-3}$ (e.g. at 05:50:15 UT). Finally, beginning at around 05:58:15 UT, a last structure shows a bridged shape, the ion energy slowly increases and then decreases. During these observations, the satellites did not encounter the magnetopause. However, the dispersed structure is probably associated with the magnetopause motion.

To unambiguously determine the origin of these low energy ions, we examined in detail the data provided by the Cluster ion spectrometer, CIS-2, and the mass spectrometer, CIS-1. Typical energy spectra beginning at 05:48:46 UT (CIS-2) and 05:48:42 UT (CIS-1) inside the first structure are displayed in Fig. 4. $\mathrm{H}^{+}, \mathrm{He}^{+}$and $\mathrm{O}^{+}$constitute the observed ion population. As evidenced in Fig. 3, at 05:48:46 UT, these ions are flowing toward the Sun and toward $+Z_{\mathrm{GSE}}$. Protons have an energy of the order of $70 \mathrm{eV} ; \mathrm{He}^{+}$ions are detected around $200-300 \mathrm{eV}$ and $\mathrm{O}^{+}$ions are detected around $1000 \mathrm{eV}$. There is a slight difference in energy between the $\mathrm{He}^{+}$peak detected by CIS- 1 and CIS-2. This is attributed to the fact that the measurements are not exactly simultaneous, that the energy of the structure is decreasing very rapidly (Fig. 3) and that the ion measurements obtained with CIS- 


\section{$31 / \mathrm{Jan} / 2001$}

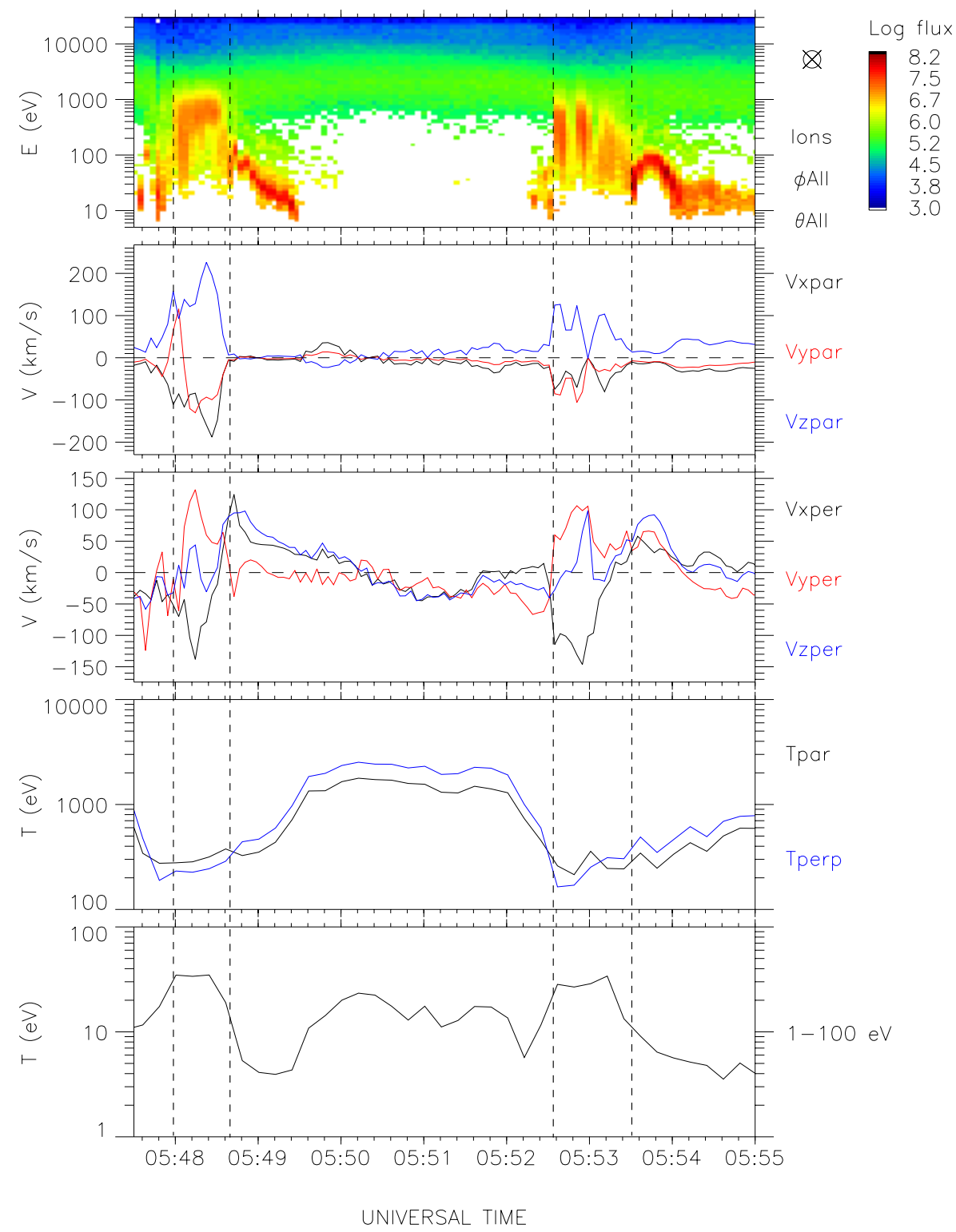

Fig. 6. Detailed moment computations for the period from 05:47:30 to 05:55:00 UT on 31 January 2001. The top panel gives the $1 \mathrm{D}$ energy-time spectrogram obtained with a time resolution of $4 \mathrm{~s}$ (all viewing directions are averaged on board). The second and third panels give the three GSE components of the ion bulk velocity parallel and perpendicular to the local magnetic field. The fourth panel displays the ion parallel and perpendicular temperatures computed in the full energy range. The last panel gives the average ion temperature computed from $5 \mathrm{eV}$ to $100 \mathrm{eV}$.
2 are averaged over $12 \mathrm{~s}$, while those obtained with CIS-1 are averaged over $32 \mathrm{~s}$. This leads to an artificial broadening in energy (temperature). However, despite these limitations, Fig. 4 clearly shows that the ion population seen after the reentry of the satellite into the magnetosphere is of ionospheric origin and that ions with different masses are all moving with the same velocity. This has been verified for all low energy structures presented in Fig. 3.

The characteristics of the motion of these ions have been determined using their 3D distribution functions. Figure 5 displays these distributions for ions without mass selection in the $\left(V_{/ /}, V_{\perp 1}\right)$ and $\left(V_{\perp 1}, V_{\perp 2}\right)$ planes. $V_{\perp 1}$ is in the $\left(V_{/ /}\right.$, $V$ bulk) plane, and $V_{\perp 2}$ is perpendicular to both $V_{/ /}$and $V_{\perp 1}$. This figure, which pertains to the energy dispersed structure encountered after the first re-entry into the magnetosphere during the period from 05:48:20 to 05:50:00 UT, clearly shows that the low temperature plasma encountered inside the magnetosphere is flowing perpendicular to the local magnetic field. Since the ions are cold and the $\boldsymbol{E} \times \boldsymbol{B}$ velocity is large compared to their thermal velocity, they appear at the same phase in the spin, i.e. for a given value of $V_{\perp 1}$. Distributions on the left panels of the figure are taken in the middle of the energy dispersed structure, while the distributions on the right are taken near the end of the structure. The observed dynamical behaviour, i.e. the decrease in energy due to a "slow" shift of the perpendicular velocity towards the origin, is strongly indicative of the adiabaticity of the motion of the ions. We verified that the observed shape 
and variations are representative of all other events displayed in Fig. 3. Note that some broadening of the distribution displayed in Fig. 5 can be due to $\mathrm{He}^{+}$and $\mathrm{O}^{+}$ions which cannot be distinguished from protons using the CIS-2 spectrometer.

The main conclusions reached from the analysis of individual ion distribution functions are substantiated and extended by detailed computations of the moments of the ion distribution functions, as exemplified in Fig. 6. Here, the top panel gives the ion energy-time spectrogram with 62 energy steps provided by the CIS-2 instrument. The onboard software sums, for each energy step, the data obtained in all measured directions during one spin (4s). The resulting 1D spectrogram allows for a better identification of fine time and energy structures when compared to spectrograms with 31 energies and 12 s resolution (see Fig. 3 ). The second and third panels of Fig. 6 display the three GSE components of the ion parallel and perpendicular velocities computed on board the spacecraft using 62 energy steps. Inside the magnetosheath, both the perpendicular and the parallel ion velocities are higher than $100 \mathrm{~km} / \mathrm{s}$. On the contrary, inside the magnetosphere, the parallel velocity of the ionospheric ions is close to zero (05:48:34-05:49 30 UT and 05:53:3005:54:00 UT). The kinetic energy variations of these ions then follows, as expected, the variations of their perpendicular velocity. Note, for example, the inverted $U$ shaped of the structure centered on 05:53:44 UT which exactly follows the variation in the $V_{Z}$ GSE component of the perpendicular velocity. This is further proof that this low energy plasma is controlled by the $\boldsymbol{E} \times \boldsymbol{B}$ drift. Note also that the satellite encounters plasma populations with different temperatures as shown in the two last panels of Fig. 6. The fourth panel gives the parallel and perpendicular temperatures computed in the full energy range. Inside the plasma sheet between 05:49:30-05:52:00 UT, the ion temperatures reach $\sim 3 \mathrm{keV}$ and display a perpendicular anisotropy $\left(T_{\perp}>T_{/ /}\right)$. Inside the magnetosheath, the ion temperatures decrease down to about $300 \mathrm{eV}$. In the regions adjacent to the magnetosheath with a mixture of ionospheric and plasma sheet ions, the temperature computed over the full energy range is no more adequate to characterize the ion populations. The fifth panel displays the ion temperature computed in the energy range of $5-100 \mathrm{eV}$, i.e. in a range adapted to the determination of the characteristics of the low energy (ionospheric) component. Here, the ion temperature is lower than $10 \mathrm{eV}$ inside the ionospheric structures. This value of the temperature is an upper limit, as it has been computed on the ground using only 31 energy steps and 3D distributions averaged over $12 \mathrm{~s}$. Individual fits of the 1D distributions displayed in Panel 1 of Fig. 6 provide numbers close to $1 \mathrm{eV}$. This clearly demonstrates that the plasma of ionospheric origin detected in layers adjacent to the magnetopause is cold. No strong heating mechanisms are acting after the plasma was expelled from the ionosphere. Note that after 05:54 UT, ionospheric plasma is detected with a parallel velocity of about $30 \mathrm{~km} / \mathrm{s}$, i.e. comparable to its perpendicular velocity (see also Fig. 3); this particular observation is reminiscent of that reported by Chandler et al. (1999) and suggests that the ions are expelled

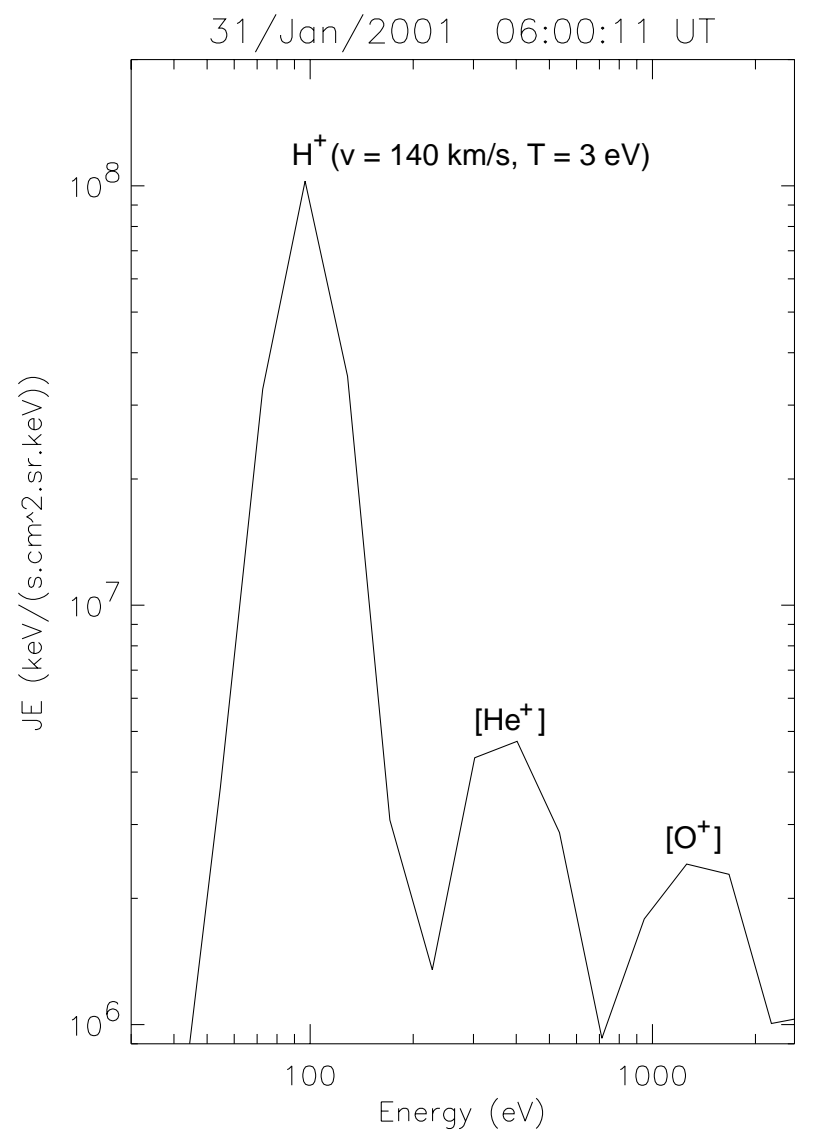

Fig. 7. Ion energy spectra taken in the plasma flow direction by the non-mass resolving CIS-2 spectrometer. Note the appearance of peaks corresponding to $\mathrm{H}^{+}, \mathrm{He}^{+}$and $\mathrm{O}^{+}$with a velocity of $\sim 140 \mathrm{~km} / \mathrm{s}$, i.e. an electric field of $\sim 2.8 \mathrm{~m} \mathrm{~V} / \mathrm{m}$. The $\mathrm{H}^{+}$temperature estimated from a maxwellian fit is close to $3 \mathrm{eV}$.

from the ionosphere by polar wind-like processes.

This ion low temperature coupled to the plasma large bulk flow allows one to easily distinguish different ions with nonmass resolving spectrometer. An example is given in Fig. 7 which pertains to the bridged structure detected around 06:00 UT in Fig. 3. The hydrogen, single ionized helium and oxygen components are here clearly apparent. The proton temperature is estimated, using a maxwellian fit to be $\sim 3 \mathrm{eV}$. The plasma velocity is $140 \mathrm{~km} / \mathrm{s}$ and using the $\boldsymbol{E}+\boldsymbol{V} \times \boldsymbol{B}=0$ relation, leads to a value of $2.7 \mathrm{~m} \mathrm{~V} / \mathrm{m}$ for the local electric field. This value is in good agreement with the $2.5 \mathrm{~m} \mathrm{~V} / \mathrm{m}$ measured by the EFW experiment (M. André, personal communication, 2001).

Other events, showing similar structures, have been identified in the Cluster data base after the mission commissioning phase. They are detected quite commonly at highlatitudes in the vicinity of the magnetopause during periods of weak magnetic activity. However, the number of dayside magnetopause crossings performed after the commissioning is limited and a complete statistical study will only be done in the future. An overview of such an event with the 


\section{$16 /$ Feb/2001}

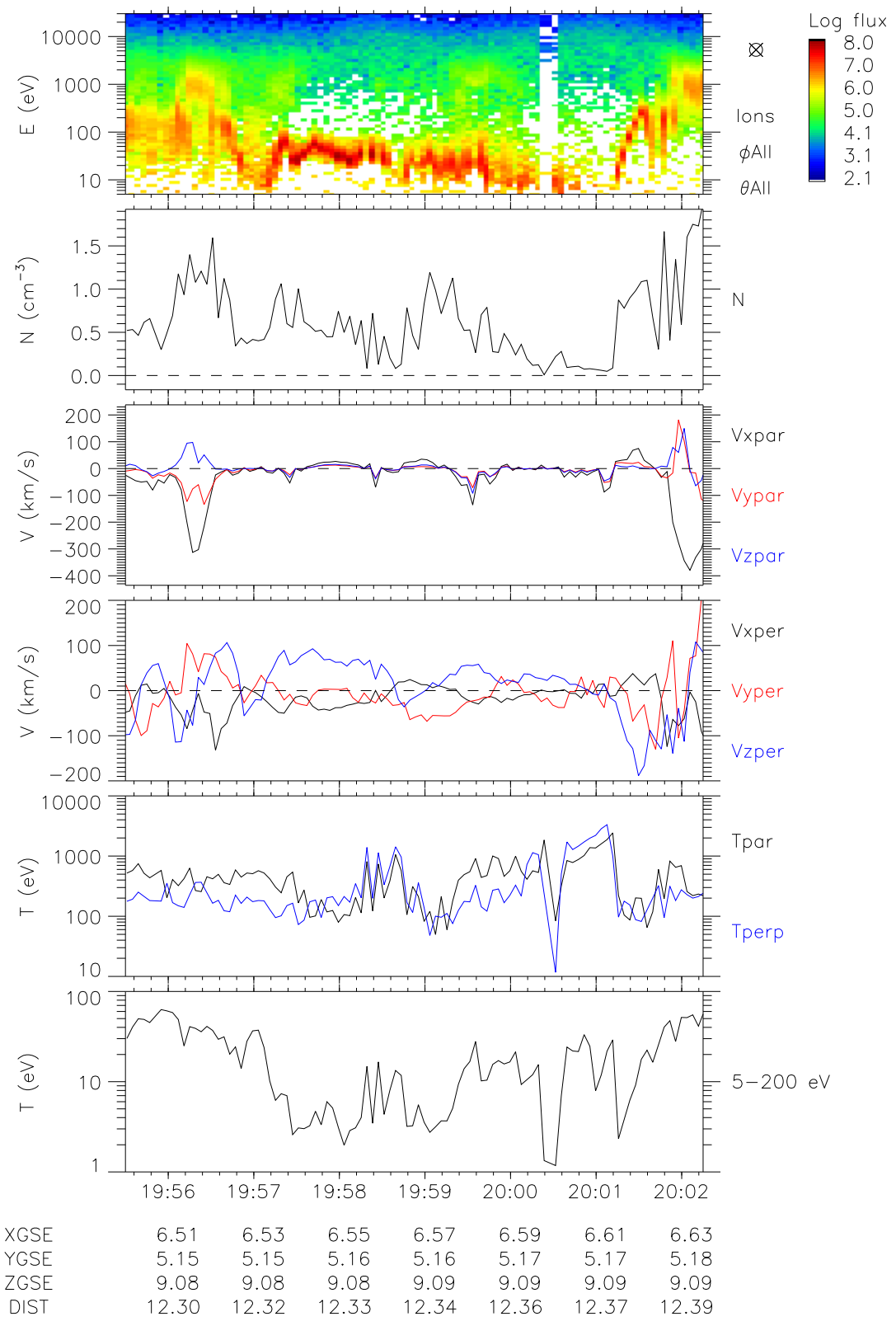

Fig. 8. Same as Fig. 6 for an event occurring on 16 February 2001 except that the density variation has been added to this figure (panel 2). main characteristic similar to the 31 January case is given in Fig. 8. The format of this figure is similar to that of Fig. 6. Magnetosheath-like plasma with energy of about $1 \mathrm{keV}$ is encountered first during a brief interval from 19:56:10 to 19 56:30 UT and then after 20:01:40 UT. The magnetosheath encounters are characterized by a strong parallel velocity of the ion bulk flow. Note that on that day (16 February 2001), the magnetosheath density is relatively small $\left(1-2 \mathrm{~cm}^{-3}\right)$. Between the two magnetosheath encounters, the satellite detects low energy ions with almost no parallel velocity. The energy variations displayed on the energy-time spectrogram follow from the variations of the ion perpendicular velocity. This is particularly clear for the two time intervals, 19:57:00-
10:58:30 UT and 20:01:30 UT. Note that the density of the ionospheric plasma is comparable to that measured in the magnetosheath. Its temperature (bottom panel) varies in the range of $\sim 2-15 \mathrm{eV}$.

\section{Discussion and summary}

On 31 January 2001, Cluster encountered the magnetopause several times during the interval between 05:30 to 06:02 UT. The Cluster satellites 1 and 3 were providing data on that day. For that period, each magnetopause crossing is accompanied by the appearance of a layer of ionospheric ions inside 


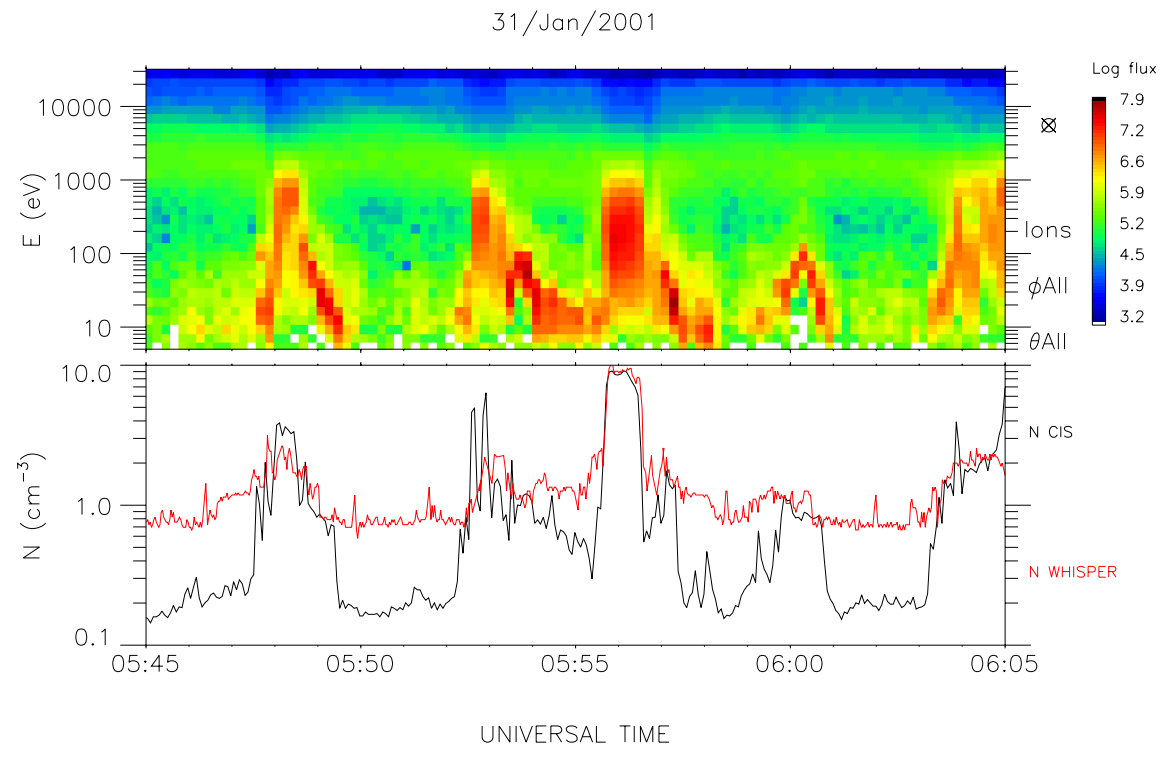

Fig. 9. Densities comparisons. Top panel, the CIS-2 energy-time spectrogram. Bottom panel, the plasma densities computed from the CIS instrument (black curve) and from the plasma frequency measured by the WHISPER experiment (red curve). Note that both curves agree only in structures where the plasma is flowing (see Fig. 3). Inside non-flowing plasma regions, the densities differ by a factor of $\sim 4$ which is attributed to the effect of the satellite potential (see text).

the magnetosphere. Plasma composition measurements indicate that the major ions are $\mathrm{H}^{+}, \mathrm{He}^{+}$and $\mathrm{O}^{+}$. No evidence for the formation of a well-defined boundary layer with ions of solar wind origin was found during the time interval of these observations. The cold ions show a repetitive pattern of energy changes. While the magnetopause is approaching the satellite, their energy increases from the detector low energy threshold up to about $100 \mathrm{eV}$ for protons. After the passage of the satellites into the magnetosheath and just following their re-entry into the magnetosphere, the ion energy decreases from about $100 \mathrm{eV}$ for protons down to the lowest detectable energy. This behaviour is interpreted due to the effect of the electric field associated with the magnetopause motions. The ion motion is set up when the magnetopause boundary is pushed inward, and relaxed when the boundary is moving out. This interpretation is substantiated by the change in the proton distribution functions, which suggests the conservation of the first adiabatic invariant in the frame moving with $\boldsymbol{E} \times \boldsymbol{B}$. In the opposite case, the ions would be accelerated without the possibility of returning to thermal energy when the magnetopause is moving far away. It must be stressed that this behaviour has been revealed for protons. For the other species, the data acquisition rate was too low to definitively reach a conclusion about adiabaticity. Furthermore, that the energization of the ions is linked to the magnetopause motion can be deduced from their velocity distribution. As exemplified by Fig. 3 (third panel from the top), low energy ions are primarily flowing tailward when the magnetopause approaches the satellite, and sunward when the magnetopause retreats. Note that when the magnetopause retreats, the ions are flowing primarily along the $+Z_{\mathrm{GSE}}$ axis. These signatures are not always associated with the magnetopause crossing by Cluster. For example, the inverted-V shape at 06:00-06:01 UT can be understood as the result of a bulk acceleration followed by a deceleration of the plasma, as the magnetopause accelerated outward and then its motion slowed down in the same direction. The drift of the thermal plasma caused by magnetopause motion allows us to determine the composition of the initially cold plasma even with the non-mass resolving CIS-2 instrument (Fig. 7). Furthermore, it allows one to measure its density which typically reaches values of the order of $1 \mathrm{~cm}^{-3}$ for this pass, i.e. about 5 times the plasma sheet density.

These measurements show that ionospheric plasma may, at times, gain enough energy perpendicular to the magnetic field near the magnetopause to overcome the satellite potential and become detectable. An important question is then natural: are the magnetopause motions revealing a background of ionospheric plasma quite broadly distributed or is the layer of ionospheric plasma closely attached to the magnetopause? A direct comparison between the CIS data and the plasma density directly deduced from the plasma frequency measured by the WHISPER experiment is presented in Fig. 9 (see Décréau et al., 1997; this issue). The answer is clear: CIS is detecting ionospheric plasma only when the magnetopause motion reveals it. This can be appreciated from the good agreement in density values found between the two experiments inside the magnetosheath and inside the attached moving layers of ionospheric plasma. However, between encounters with the magnetosheath, the plasma density directly computed from the plasma frequency stays at a level of about $1 \mathrm{~cm}^{-3}$, while the density deduced from CIS falls below $0.2 \mathrm{~cm}^{-3}$. In other words, the temperature of the plasma is too weak to allow it to overcome the satellite potential. From the distribution function of the ions close to the magnetopause, we found that the transition between plasma from the magnetosheath and plasma from the ionosphere can be very fast, corresponding to one satellite spin period, i.e. about $600 \mathrm{~km}$ for a magnetopause moving with a velocity of $150 \mathrm{~km} / \mathrm{s}$.

A detailed multi-experiment determination of the global distribution of this ionospheric plasma inside the dayside and 
nightside magnetosphere of spatial scales, velocity gradients and of the general implications of this plasma population originating from the ionosphere will be presented in forthcoming papers.

Acknowledgements. The authors are pleased to acknowledge the technical teams at CESR/CNRS, Toulouse; UNH, Durham; IFSI, Frascati; UCB, Berkeley; MPI Garching; MPAE, Lindau; UW, Seattle; and ISP, Kiruna; for their invaluable contribution of performing long and exacting job of defining, assembling, and calibrating the CIS instruments for the Cluster missions, under the leader ship of C. Aoustin, CIS Project Manager. The data display software has been performed by E. Penou and A. Barthe. The work at CESR is supported by CNES (French Space Agency). The authors thank the referees for their useful comments.

Topical Editor G. Chanteur thanks two referees for their help in evaluating this paper.

\section{References}

Balogh, A., Dunlop, M. W., and Cowley, S. W. H.: The Cluster magnetic field investigation, Space Science Reviews, 79, 65, 1997.

Burch, J. L., Mitchell, D. G., Sandel, B. R. Brandt, P. C., and Wüest, M.: Global dynamics of the plasmasphere and ring current during magnetic storms, Geophys. Res. Lett., 6, 1159, 2001.

Carlson, C., Curtis, D. W., Paschmann, G., and Michael, W.: An instrument for rapidly measuring plasma distribution functions with high resolution, Adv. Space Res., 2, 67, 1982.

Carlson, C. W. and McFadden, J. P.: Design and application of imaging plasma instruments, in: "Measurements techniques in space plasma", (Eds) Pfaff, R. F., Borovsky, J. E., and Young, D. S., AGU Geophysical monograph, 102, pp. 125-140, 1998.

Carpenter, D. L. and Anderson, R. R.: An ISEE/Whistler model of equatorial electron density in the magnetosphere, J. Geophys. Res., 97, 1097, 1992.

Chandler, M. O., Fuselier, S. A., Lockwood, M., and Moore, T. E.: Evidence of component merging equatorward of the cusp, J. Geophys. Res., 104, 22 623, 1999.

Chappell, C. R., Moore, T. E., and Waite, Jr., J. H.: The ionosphere as a fully adequate source of plasma for the Earth's magnetosphere, J. Geophys. Res., 92, 5896, 1987.

Chen, A. J., Grebowsky, J. M., and Taylor, Jr., H. A.: Dynamics of the mid-latitude light ion trough and plasma tails, J. Geophys. Res., 80, 968, 1975.

Décréau, P. M. E., Fergeau, P., and Krasnnosels'kikh, V., et al.: Whisper, a resonance sounder and wave analyzer, Performances and perspectives for the Cluster mission, Space Science Review, 79, $157,1997$.

Delcourt, D. C., Sauvaud, J-A., and Moore, T. E.: Polar wind ion dynamics in the magnetotail, J. Geophys. Res., 98, 9155, 1993.

Delcourt, D. C., Chapell, C. R., Moore, T. E., and Waite, Jr., J. H.: A three dimensional numerical model of ionospheric plasma in the magnetosphere, J. Geophys. Res., 94, 11 893, 1989.
Fuselier, S. A., Klumpar D. M., Peterson, W. K., and Shelley, E. G.: Direct injection of ionospheric $\mathrm{O}^{+}$into the dayside low latitude boundary layer, Geophys. Res. Lett., 16, 1121, 1989.

Lundin, R. and Dubinin, E.: Solar wind energy transfer regions inside the dayside magnetopause - Accelerated heavy ions as tracers for MHD-processes in the dayside boundary layer, Planet. Space Sci., 33, 891, 1985.

McFadden J. P. and Carlson, C. W.: Computer simulation in designing electrostatic optics for space plasma experiments, in: "Measurements techniques in space plasma", (Eds) Pfaff, R. F., Borovsky, J. E., and Young, D. S., AGU Geophysical monograph, 102, pp. 249-256, 1998.

Möbius, E., Kistler, L. M., and Popecki, et al.: The 3-D plasma distribution function analyzers with time-of-flight mass discrimination for Cluster, Fast, and Equator-S, in: "Measurements techniques in space plasma", (Eds) Pfaff, R. F., Borovsky, J. E., and Young, D. S., AGU Geophysical monograph, 102, pp. 243-248, 1998.

Moore, T. E., Chappell, C. R., and Chandler, M. O., et al.: The thermal ion dynamics experiment and plasma source instrument, Space Science Reviews, 71, 409, 1995.

Rème, H., Bosqued, J.-M., and Sauvaud, J.-A., et al.: The Cluster ion Spectrometry (CIS) experiment, Space Science Reviews, 79, 303, 1997.

Rème, H., Bosqued, J.-M., and Lavraud, B.: First mulftispacecraft ion measurements in and near the Earth's magnetosphere with the identical Cluster ion spectrometry (CIS) experiment, Ann. Geophysicae, this issue, 2001

Riedler, W., Torkar, K., and Rüdenauer, F., et al., Active spacecraft potential control, Space Science Reviews, 79, 271, 1997.

Sauvaud, J.-A. and Delcourt, D. C.: A numerical study of suprathermal ion trajectories in three-dimensional electric and magnetic field models, J. Geophys. Res., 92, 5873, 1987.

Shelley, E. G., Johnson, R. G., and Sharp, R. D.: Satellite observations of an ionospheric acceleration mechanism, Geophys. Res. Lett., 3, 654, 1976.

Shelley, E. G., Ghielmetti, A. G., and Balsiger, H., et al.: The toroidal imaging mass-angle spectrograph (TIMAS) for the Polar mission, Space Science Reviews, 71, 497, 1995.

Shue, J.-H., Chao, J. C., Russell, C. T., Song, P., Khurara, K. K., and Singer, H. P.: A new functional form to study the solar wind control of the magnetospause size, J. Geophys. Res., 102, 94, 967, 1997.

Su, Y. J., Borovsky, J. E., Thomsen, M. F., Elphic, R. C., and McComas, D. J.: Plasmaspheric material on high-latitude open field lines, J. Geophys. Res., 105, 7591, 2000.

Su, Y. J., Borovsky, J. E., Thomsen, M. F., Dubouloz, N., Chandler, M. O., Moore, T. E., and Bouhram, M.: Plasmaspheric material on high-latitude open field lines, J. Geophys. Res., 106, 6085, 2001.

Yau, A. W. and André, M.: Sources of ion outflow in the high latitude ionosphere, in: "Transport Across the Boundaries of the Magnetosphere", (Eds) Hultqvist, B. and Oieroset, M., Kluwer Academic press, Dordrecht, pp. 1-25, 1997. 